\title{
Modelling and variability of selected chemical properties of shallow water wells using GIS in Wasit and Dhi Qar provinces/Iraq
}

\author{
Basima Abbas Jabir Al-humairi', Nadhum Shamkhi Rahal ${ }^{2}$ and Ahmed Kareem Jebur ${ }^{3}$ \\ ${ }^{1}$ Department of Water Resources Techniques, Kut Technical Institute, Middle Technical University, \\ Baghdad, Iraq \\ ${ }^{2}$ Department of Plant Production, Suwaira Technical Institute, Middle Technical University, Baghdad, Iraq \\ ${ }^{3}$ Department of Surveying Techniques, Kut Technical Institute, Middle Technical University, Baghdad, Iraq \\ [Received: October 27, 2019 Accepted: December 10, 2019 Published Online: February 28, 2020]
}

\section{Abstract}

This study was conducted to model several chemical properties of shallow well waters and to investigate the spatial variability of these properties in different regions over two provinces in Iraq - Wasit and Dhi-Qar. Eleven wells were chosen to evaluate the quality of their water and its suitability for agricultural, civil engineering and human applications and uses. Samples were taken in January 2018 from Giazna (1, 2, 3, and 4), Karthiya, Badra, Dujaili, Shaikhsaad, Muwafaqiya, Hay, in Wasit province and from Fajr in Dhi-Qar province. The water samples were subjected to chemical analysis to measure the concentrations of sodium $\left(\mathrm{Na}^{1+}\right)$, calcium $\left(\mathrm{Ca}^{2+}\right)$, magnesium $\left(\mathrm{Mg}^{2+}\right)$, $\mathrm{pH}$, electrical conductivity (Ec), and total dissolved solids (TDS). Results were undergone several descriptive statistics Minimum (Min.), Maximum (Max.), standard deviation (std), skewness (skew.), and Kurtosis, (Kurt.) and coefficient of variation (CV\%). Mathematical models were generated using Data Fit (9.3) software to predict sodium adsorption ratio (SAR) and exchangeable sodium percentage (ESP) depending on chemical properties. The predicted values of SAR and ESP were validated using several validation indices including the factor of determination $R^{2}$, root mean squared error (RMSE), absolute mean error (MAE), relative error (RE), correlation coefficient (r) and standard error (s). A GIS program was used to map the studied chemical properties. The results showed that the SAR model $\left(S A R=a_{1} \times \mathrm{Na}^{1+}+a_{2} \times \mathrm{Ca}^{2+}+a_{3} \times\right.$ $M^{2+}+a_{4} \times E S P+a_{5} \times X$ east $+a_{6} \times Y$ north $\left.+a_{7}\right)$ which with $\left(R^{2}=0.999, S E E=5.30, R M S E=0.000, M A E=0.000\right.$, and $R E=0.000)$ and the ESP model $\left(E S P=E x p\left(a_{1} \times \mathrm{Na}^{1+}+a_{2} \times \mathrm{Ca}^{2+}+a_{3} \times \mathrm{Mg}^{2+}+a_{4} \times X\right.\right.$ east $+a_{5} \times Y$ north $\left.\left.+a_{6}\right)\right)$ which with $\left(R^{2}=0.9827, S E E=5.55, R M S E=0.037, M A E=0.026\right.$ and $\left.R E=0.003\right)$ were the best for predicting $S A R$ and ESP values for the selected wells. The Well water quality index (WWQI) was determined and accordingly the water was classified as poor. As a result, the water of the studied wells was considered unsuitable for human consumption as drinking water and irrigation; except the well of Fajr which was found critically suitable for irrigation purposes.

Keywords: WWQI, SAR, ESP, GIS, coefficient of variability, validation indices

\section{Introduction}

Water is a vital component for all forms of life and is the most important factor in shaping the land and regulating the climate (Durgadevagi et al., 2016). The extreme shortage of water resources in general and lack of surface water resources in specific has caused an increasing need for suitable water for human and agricultural uses. And its scarcity is one of the main problems of sustainable agriculture that compelled farmers for extensive use of other water resources for food production (Moghabel et al., 2017; Sabeen et al., 2020). Thus, there has been an urgent need to look for alternative natural water recourses.

One of the uppermost water resources in rural areas is groundwater. The suitability of groundwater for drinking, industrial, domestic, agricultural and other purposes in arid and semi-arid regions have been a major challenge worldwide (Barzegar et al., 2016; Durgadevagi, 2016; Narasimha et al., 2018; Abd El-Aziz, 2018). Also, it becomes a significant and crucial component for all development activities of any life support system and one of the fundamental sources of water for drinking, irrigation and industrial use in many countries (Mohamed et al., 2019). And its pollution has become a threat to various usages (Hussain et al., 2015).

In recent years, stress on natural resources has been increasing due to rapid industrialization and growth in population; and hence the conservation of such resources is one of the major challenges to mankind nowadays (Abbasnia et al., 2018). So, the quality of groundwater resources should be taken into full considerations

\footnotetext{
*Email: nadhum_rl@yahoo.com
} 
(Aghazadeh and Moghaddam, 2010). The analysis of groundwater quality is very important to preserve and protect the natural ecosystem and it is controlled by several factors including rock type, climate and human activities (Elhag, 2016). The evaluation of groundwater quality is paramount to arid and semi-arid regions (Bhat et al., 2016). It is important to monitor and evaluate the quality of groundwater that may be adequate to be used for various purposes (Barzegar and Moghaddam, 2016). Groundwater degradation can be caused by natural and anthropogenic activities and processes in addition to the improper waste disposal of the surface bodies. Consequently, the groundwater is subjected to various sources of contaminations (Barzegar and Moghaddam, 2016; Durgadevagi et al., 2016) and hence bad water quality may cause health problems. Continues monitoring of the quality of water is very essential, and the collected data will be crucial to develop effective strategies for improving rural drinking water supply and provide evidence for effective decisions and management for groundwater; and also to propose an approach that can guarantee reliable findings (Narsimha et al., 2018; Mohamed et al. 2019; Khalid, 2019). The quality of groundwater is also influenced by the mineralogy of the aquifer and recharge pathways (Wali, et al., 2018). Groundwater quality is usually characterized by different physical and chemical properties. Its change varies widely due to the seasonal fluctuation of rain, various types of contamination and depletion of groundwater. Hence, its quality evaluation over space and time proved to be an important technique for solving different hydrochemistry issues and it is important for effective utilization and development of this finite resource (Wali et al., 2018).

Several studies have considered the quality of wells waters (Faozi and Aziz, 2006; Hassan and Mohammed, 2005; Yasin, 2009; Adnan and Yusra, 2017; Hussain and Rajab, 2009). They, however, were limited and did not address the spatiotemporal variability of chemical properties of shallow well waters. So, the current study has been conducted to model selected chemical properties of several well waters and to calculate water quality index (WWQI) for them and then representing their variability and patterns of distribution cartographically using GIS technique.

\section{Materials and Methods}

\section{Study area}

The two study areas are situated in Wasit and Dhi-Qar provinces (Figure 1). The land of provinces is part of the Mesopotamian plain. Their landscape is characterized by a plane surface with few undulating surfaces due to the ancient irrigation system. Their soils were mostly driven from river alluvium. The prevailing climate of the two provinces is very dry and hot in summer and with few showers in winter (less than $100 \mathrm{~mm} /$ year). The people of this area depend mainly on agricultural activities, and the main cultivated crops are cereals and summer vegetables with few gardens assigned for date palms. The main source of water in the area is the Tigress river and its branch AlGharaf. However, in recent years and due to the low water discharges coming from the river and its tributaries especially in the summer season there has been a critical shortage of irrigation water for the crops. As a result, farmers turned to use hand-dug shallow wells for their daily consumption and to irrigate their lands. Unfortunately, they have been using the well water without applying it to any standardized test.

\section{Sampling and Laboratory analysis}

Samples of groundwater were collected from eleven shallow hand-dug boreholes located in selected areas within Wasit and Dhi-Qar provinces (Figure 1). The coordinates and location details were determined using a GPS instrument and are listed in Table 1. Water samples were subjected to chemical analysis using standard analytical methods (Richards 1954) to measure their chemical properties such as $\mathrm{pH}, \mathrm{EC}, \mathrm{Ca}^{2+}, \mathrm{Mg}^{2+}, \mathrm{Na}^{1+}$, and TDS. The instruments and apparatus used were $\mathrm{pH}$ meter type Andwa: AD1200, EC metre type: YSI,556 mps, Flame photometer, type $\mathrm{PG}$ and versenate titration method for measuring $\mathrm{pH}$, $\mathrm{EC}, \mathrm{Na}^{1+}, \mathrm{Ca}^{2+}$, and $\mathrm{Mg}^{2+}$, respectively. The analyses were carried out in the chemical analytical laboratories of the Environmental Directorate and Agricultural Division of Wasit province. Exchangeable sodium percentage (ESP) and Sodium adsorption ratio (SAR) were calculated for all well waters using equations 1 and 2, respectively, and the results are documented in Table (1).

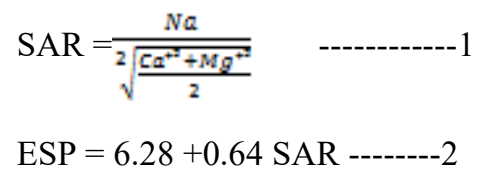

\section{Statistical spatial analysis}

Data of the chemical properties of the well water samples was subjected to descriptive statistical analysis (standard deviation, the coefficient of variation, skewness and kurtosis) employing SPSS software (version 20) as detailed in Table (1). The working sequence steps of descriptive statistics are as follows: 


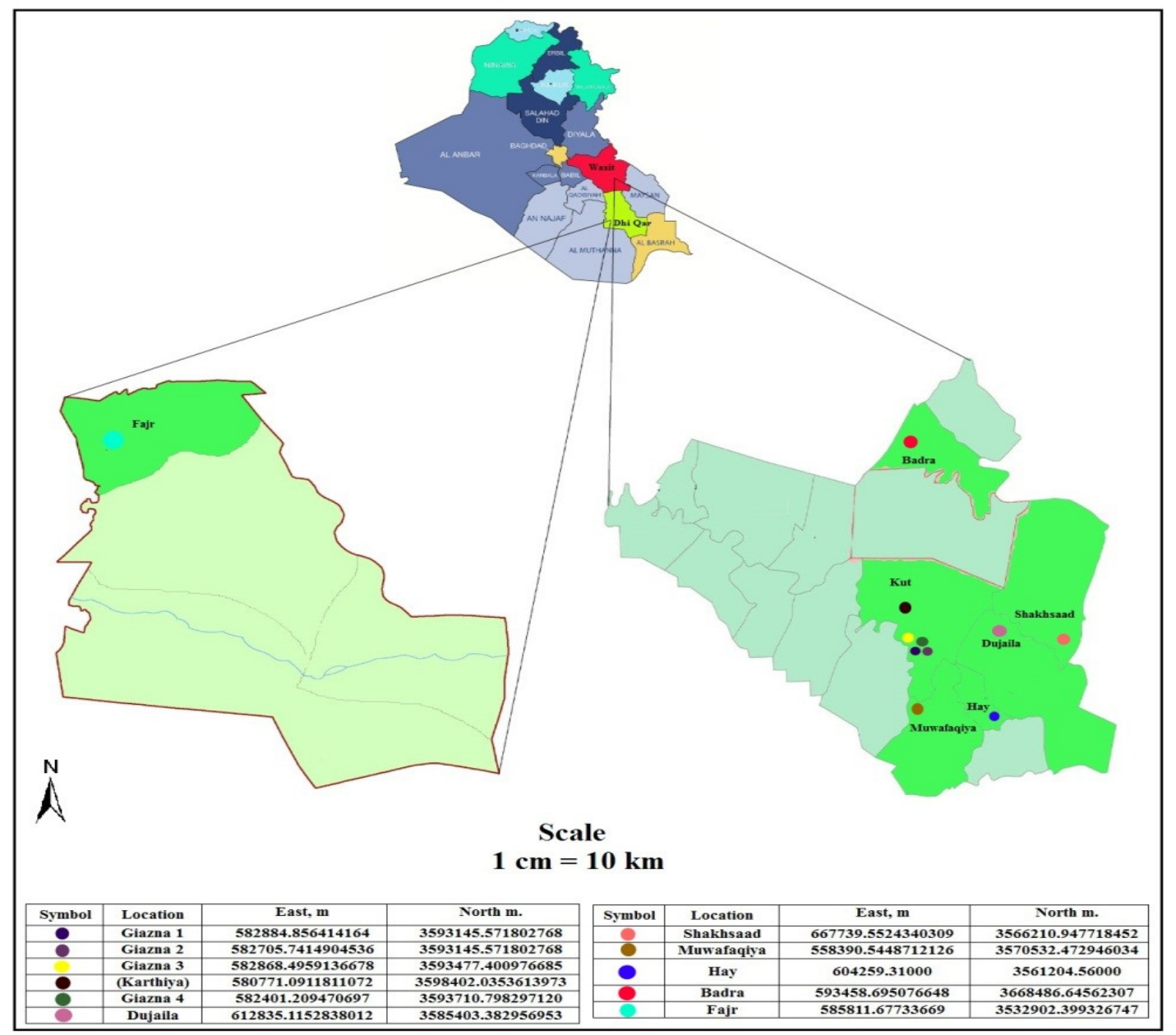

Figure 1: Study area and wells locations

Table 1: The descriptive statistics of spatial and chemical parameters of well waters

\begin{tabular}{|c|c|c|c|c|c|c|c|c|c|c|}
\hline $\begin{array}{c}\text { Parameter } \\
\text { Region }\end{array}$ & $\mathrm{X}$ East, $\mathrm{m}$ & Y North $\mathrm{m}$ & $\begin{array}{c}\mathrm{Na}^{{ }^{+}} \\
\mathrm{meq}^{-1} \mathbf{L}^{-1}\end{array}$ & $\begin{array}{c}\mathrm{Ca}^{2+} \\
\mathrm{meq} \mathrm{L}^{-1}\end{array}$ & $\begin{array}{c}\mathbf{M g}^{2+} \\
\text { meq L L }^{-1}\end{array}$ & $\begin{array}{c}\text { EC } \\
\text { dS m }^{-1}\end{array}$ & pH & $\begin{array}{c}\text { TDS } \\
\mathrm{mg} \mathrm{L}^{-1}\end{array}$ & SAR & ESP\% \\
\hline Giazna 1 & 582884.856414164 & 3593145.571802768 & 5.739 & 5.131 & 3.13 & 1.400 & 7.700 & 896.000 & 2.824 & 8.087 \\
\hline Giazna 2 & 582705.7414904536 & 3593145.571802768 & 6.434 & 7.506 & 4.060 & 1.800 & 7.600 & 1152.000 & 2.675 & 7.992 \\
\hline Giazna 3 & 582868.4959136678 & 3593477.400976685 & 8.260 & 9.162 & 5.578 & 2.500 & 7.700 & 1600.000 & 2.855 & 8.107 \\
\hline (Karthiya) & 580771.0911811072 & 3598402.0353613973 & 6.870 & 11.050 & 3.082 & 2.290 & 6.250 & 1465.600 & 2.584 & 7.934 \\
\hline Giazna 4 & 582401.209470697 & 3593710.798297120 & 11.260 & 10.208 & 11.532 & 3.300 & 7.800 & 2112.000 & 3.415 & 8.466 \\
\hline Dujaila & 612835.1152838012 & 3585403.382956953 & 5.435 & 8.700 & 2.499 & 1.790 & 6.360 & 1145.600 & 2.297 & 7.750 \\
\hline Shakhsaad & 667739.5524340309 & 3566210.947718452 & 6.000 & 9.600 & 2.749 & 1.981 & 6.460 & 1267.840 & 2.415 & 7.826 \\
\hline Muwafaqiya & 558390.5448712126 & 3570532.472946034 & 4.783 & 8.000 & 2.332 & 1.630 & 6.450 & 1043.200 & 2.104 & 7.627 \\
\hline Hay & 604259.31 & 3561204.56 & 6.087 & 9.750 & 2.748 & 2.020 & 6.460 & 1292.800 & 2.435 & 7.838 \\
\hline Badra & 593458.695076648 & 3668486.64562307 & 13.391 & 16.504 & 10.105 & 4.000 & 7.600 & 2560.000 & 3.671 & 8.629 \\
\hline Fajr & 585811.67733669 & 3532902.399326747 & 5.173 & 3.725 & 3.102 & 1.200 & 7.800 & 768.000 & 2.799 & 8.071 \\
\hline Min. & & & 4.780 & 3.735 & 2.332 & 1.200 & 6.250 & 768.000 & 2.104 & 7.627 \\
\hline Max. & & & 13.390 & 16.50 & 11.532 & 4.000 & 7.800 & 2560.000 & 3.671 & 8.629 \\
\hline Mean & & & 7.221 & 9.031 & 4.628 & 2.170 & 7.11 & 1391.180 & 2.734 & 8.030 \\
\hline Std & & & 2.730 & 3.302 & 3.205 & 0.831 & 0.686 & 532.158 & 0.466 & 0.298 \\
\hline CV\% & & & 37.800 & 36.560 & 69.240 & 38.25 & 9.660 & 38.250 & 17.050 & 3.71 \\
\hline Skew. & & & 1.590 & 0.728 & 1.664 & 1.252 & -.209 & 1.252 & 0.890 & 0.900 \\
\hline Kurt. & & & 1.741 & 2.263 & 1.499 & 1.254 & -.337 & 1.254 & 0.487 & 0.485 \\
\hline
\end{tabular}


Start $>$ file $>$ new $>$ worksheet $>$ input data $>$ analyze $>$ statistics $>$ descriptive statistics $>$ tick the required statistics $>\mathrm{OK}>$ output data save the file or print

Pearson correlation tests $(\rho=0.05$ and $\rho=0.01)$ were performed using SPSS software to examine the strength of the inter-relationships between water chemical parameters that may help in model constructing that served for estimating SAR and ESP, Table (3). The working steps sequence of correlation is as follows:

Start $>$ file $>$ new $>$ worksheet $>$ input data $>$ analyze $>$ correlation/ bivariate $>$ choose the attribute needed $>$ tick the appropriate method of correlation $>$ flag significant $>$ OK > output data correlation matrix or print

Table 2 -A: Water quality index of waters of studied wells for human purposes

\begin{tabular}{|c|c|c|c|c|c|c|c|c|c|c|}
\hline Parameter & & $\mathrm{pH}$ & $\mathrm{EC} \mathrm{dS} \mathrm{m^{-1 }}$ & TDS mg L ${ }^{-1}$ & Na meq $\mathrm{L}^{-1}$ & Ca meq $\mathrm{L}^{-1}$ & Mg meq $\mathrm{L}^{-1}$ & SAR & ESP\% & $\begin{array}{l}\text { WWQI } \\
\sum W i * q i\end{array}$ \\
\hline Purpose & Well & & & & & & & & & $\sum W i$ \\
\hline \multirow{11}{*}{ Human } & Giazna 1 & 13.69 & 140.00 & 0.09 & 7.59 & 5.13 & 18.04 & - & - & \\
\hline & Giazna 2 & 13.51 & 180 & 0.12 & 8.51 & 7.51 & 23.40 & - & - & 146.62 \\
\hline & Giazna 3 & 13.69 & 250 & 0.16 & 10.92 & 9.16 & 32.15 & - & - & 198.87 \\
\hline & Karthiya & 11.11 & 229 & 0.15 & 9.08 & 11.05 & 17.77 & - & - & 175.01 \\
\hline & Giazna 4 & 13.87 & 330 & 0.21 & 14.89 & 10.21 & 66.48 & - & - & 274.10 \\
\hline & Dujaili & 11.31 & 179 & 0.11 & 7.19 & 8.7 & 14.41 & - & - & 138.86 \\
\hline & Shakhsaad & 11.48 & 198.1 & 0.13 & 7.93 & 9.6 & 15.85 & - & - & 152.94 \\
\hline & Muwafaqiya & 11.47 & 163 & 0.1 & 6.33 & 8 & 13.44 & - & - & 127.30 \\
\hline & Hay & 11.48 & 202 & 0.13 & 8.05 & 9.75 & 15.84 & - & - & 155.56 \\
\hline & Badra & 13.51 & 400 & 0.26 & 17.71 & 16.5 & 58.25 & - & - & 318.50 \\
\hline & Fajr & 13.87 & 120 & 0.08 & 6.84 & 3.73 & 17.88 & - & - & 102.17 \\
\hline
\end{tabular}

Table 2-B: Water quality index of waters of studied wells for agricultural purposes

\begin{tabular}{|c|c|c|c|c|c|c|c|c|c|c|}
\hline \multicolumn{2}{|c|}{ Parameter } & $\mathrm{pH}$ & $\mathrm{EC} \mathrm{dS} \mathrm{m^{-1 }}$ & TDS mg L L & Na meq $L^{-1}$ & Ca meq $\mathrm{L}^{-1}$ & Mg meq $\mathrm{L}^{-1}$ & SAR & ESP\% & \multirow{2}{*}{$\begin{array}{c}\text { WWQI } \\
\frac{\sum W i * q i}{\sum W i}\end{array}$} \\
\hline Purpose & Well & & & & & & & & & \\
\hline \multirow{11}{*}{ Irrigation } & Giazna 1 & 13.69 & 140.00 & 0.09 & 7.59 & 5.13 & 18.04 & 26.41 & 11.54 & 110.44 \\
\hline & Giazna 2 & 13.51 & 180 & 0.12 & 8.51 & 7.51 & 23.40 & 25.02 & 11.41 & 133.75 \\
\hline & Giazna 3 & 13.69 & 250 & 0.16 & 10.92 & 9.16 & 32.15 & 26.70 & 11.57 & 175.89 \\
\hline & Karthiya & 11.11 & 229 & 0.15 & 9.08 & 11.05 & 17.77 & 24.17 & 11.33 & 155.68 \\
\hline & Giazna 4 & 13.87 & 330 & 0.21 & 14.89 & 10.21 & 66.48 & 31.94 & 12.08 & 238.09 \\
\hline & Dujaili & 11.31 & 179 & 0.11 & 7.19 & 8.7 & 14.41 & 21.48 & 11.06 & 125.70 \\
\hline & Shaikhsaad & 11.48 & 198.1 & 0.13 & 7.93 & 9.6 & 15.85 & 22.59 & 11.17 & 137.41 \\
\hline & Muwafaqiya & 11.47 & 163 & 0.1 & 6.33 & 8 & 13.44 & 19.68 & 10.89 & 115.60 \\
\hline & Hay & 11.48 & 202 & 0.13 & 8.05 & 9.75 & 15.84 & 22.7 & 11.19 & 139.58 \\
\hline & Badra & 13.51 & 400 & 0.26 & 17.71 & 16.5 & 58.25 & 34.33 & 12.32 & 274.42 \\
\hline & Fajr & 13.87 & 120 & 0.08 & 6.84 & 3.73 & 17.88 & 26.18 & 11.52 & 99.31 \\
\hline
\end{tabular}

Table 3: Correlation matrix of studied spatial and chemical parameters

\begin{tabular}{|c|c|c|c|c|c|c|c|c|c|c|}
\hline & $\mathbf{X}$ & $\mathbf{Y}$ & $\mathbf{N a}$ & $\mathbf{C a}$ & Mg & EC & pH & TDS & SAR & ESP \\
\hline $\mathrm{X}$ & 1.00 & & & & & & & & & \\
\hline $\mathrm{Y}$ & -.136 & 1.000 & & & & & & & & \\
\hline $\mathrm{Na}$ & -.083 & $0.795 * *$ & 1.000 & & & & & & & \\
\hline $\mathrm{Ca}$ & 0.148 & $0.797 * *$ & $0.774 * *$ & 1.000 & & & & & & \\
\hline $\mathrm{Mg}$ & -.171 & $0.633^{*}$ & $0.947 * *$ & 0.581 & 1.000 & & & & & \\
\hline $\mathrm{Ec}$ & -.009 & $0.800 * *$ & $0.968 * *$ & $0.891 * *$ & $0.881 * *$ & 1.000 & & & & \\
\hline $\mathrm{pH}$ & 0.218 & 0.218 & 0.455 & -0.168 & 0.576 & 0.232 & 1.000 & & & \\
\hline TDS & -.009 & $0.800 * *$ & $0.968 * *$ & $0.891 * *$ & $0.881 * *$ & $1.000 * *$ & 0.232 & 1.000 & & \\
\hline SAR & -.165 & $0.653^{*}$ & $0.906 * *$ & 0.475 & $0.904 * *$ & $0.776 * *$ & $0.732 *$ & $0.776 * *$ & 1.000 & \\
\hline Esp & -.165 & $0.653 *$ & $0.907 * *$ & 0.475 & $0.905 * *$ & $0.776 * *$ & $0.776^{*}$ & $0.776 * *$ & $0.999 * *$ & 1.000 \\
\hline
\end{tabular}

* significant at $(\rho=0.05),{ }^{*}$ significant at $(\rho=0.01)$, Pearson correlation, $X$ (easting), $y$ (northing). 

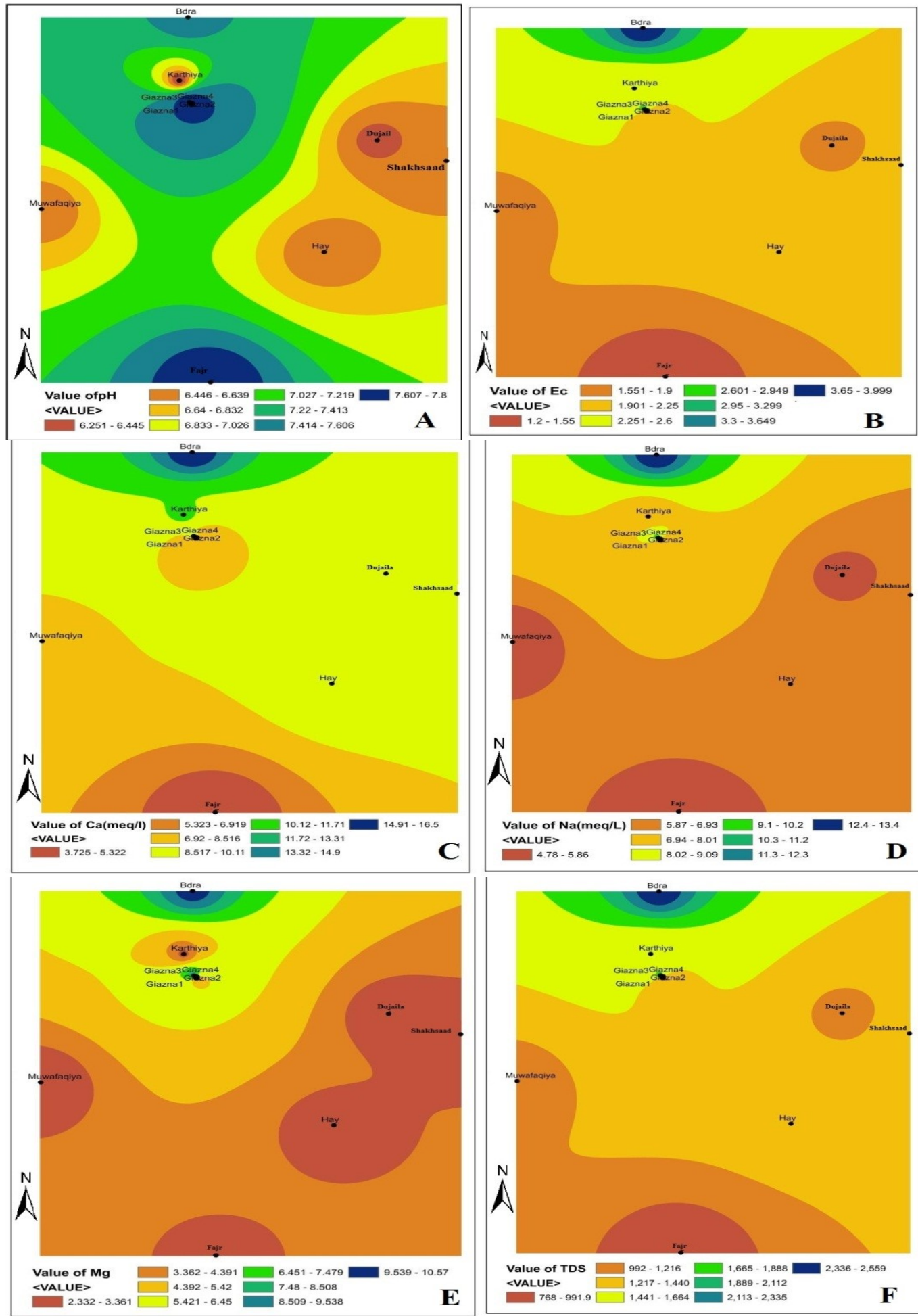

Figure 2: Spatial patterns of distribution of A (pH), B (EC), C (Ca), D (Na), E (Mg), F (TDS) 


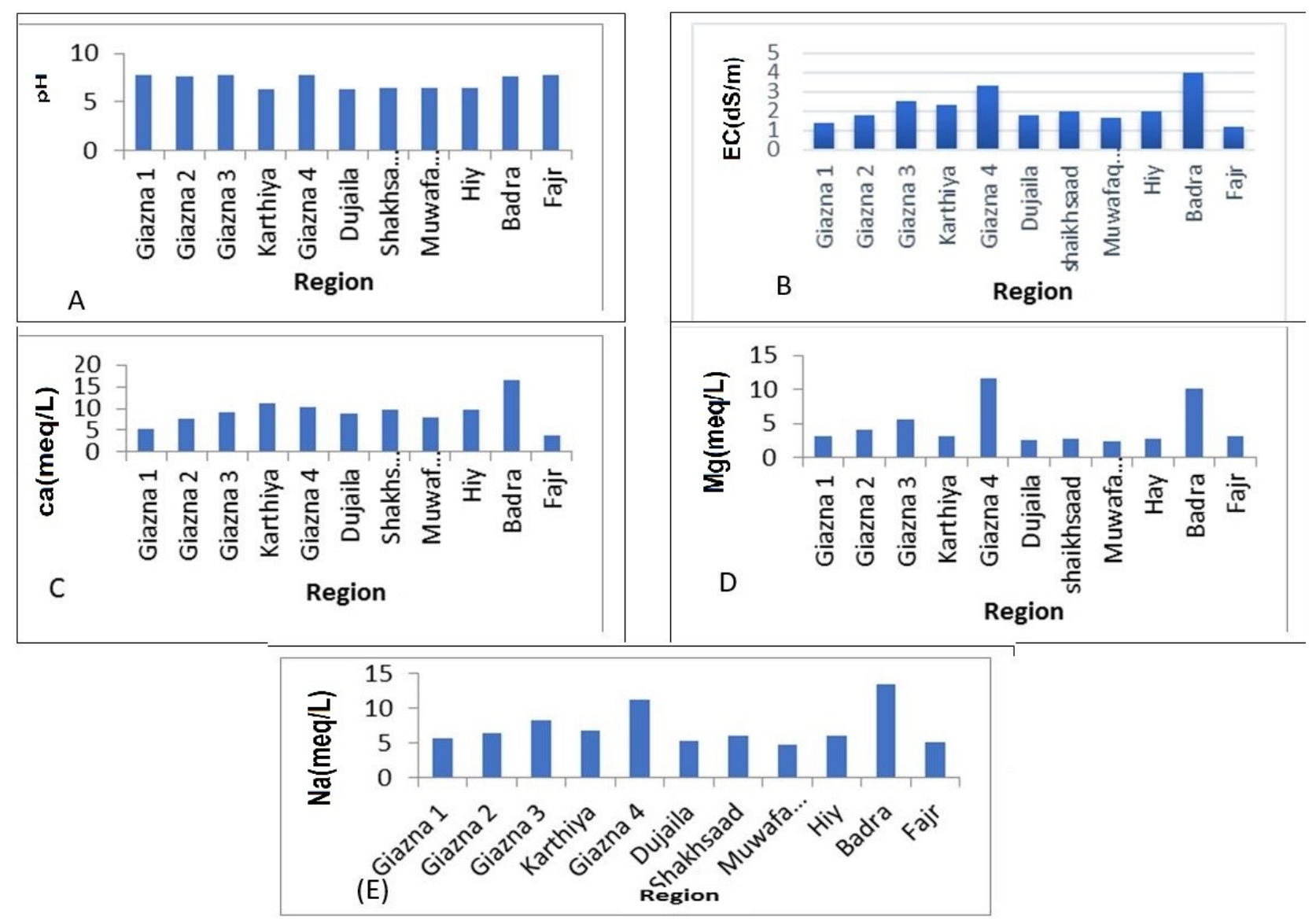

Figure 3: Represent the value of A (pH), B (EC), C (Ca), D (Mg), E (Na) to their geographical location and accordingly due to human, agricultural and anthropogenic processes and the seasonal variability of rainfall

\section{Spatial distributions and variability of the chemical properties of well waters}

Patterns of spatial variability were tested by computing minimum, maximum, mean, standard deviation, skewness, kurtosis and coefficient of variation for the chemical properties of the well water samples utilizing SPSS software, Table (1). GIS software was thereafter employed to map the spatial patterns of distribution of these properties as shown in Figure $2(\mathrm{~A}, \mathrm{~B}, \mathrm{C}, \mathrm{D}, \mathrm{E}$, and F). The coefficients of variation $(\mathrm{CV} \%)$ for all the chemical properties of water samples calculated using formula 3 and histograms were sketched to show the spatial variability and dependency of $\mathrm{pH}, \mathrm{EC}, \mathrm{Ca}^{2+}, \mathrm{Mg}^{2+}, \mathrm{Na}^{1+}, \mathrm{SAR}$ and ESP as shown in Figure 3 (A, B, C, D, and E).
$\mathrm{CV} \%=(\sigma \div$ mean $) 100$

Where: $\mathrm{CV}=$ coefficient of variation, $\sigma=$ standard of variation.

\section{Well waters quality index (WWQI):}

Water quality index was calculated using equations 4 , 5 , 6, and 7, respectively, Table ( $2 \mathrm{~A}$ and $2 \mathrm{~B})$. The water quality index pattern of distribution was mapped using GIS technique as illustrated in Figure 4

$\mathrm{qi}=\left(\mathrm{C}_{\mathrm{i}} \div \mathrm{S}_{\mathrm{i}}\right) \times 100$
$\mathrm{Wi}=1 \div \mathrm{Si}$


Where: $\mathrm{WI}=$ the relative weight of each Parameter, $\mathrm{q} i=$ the

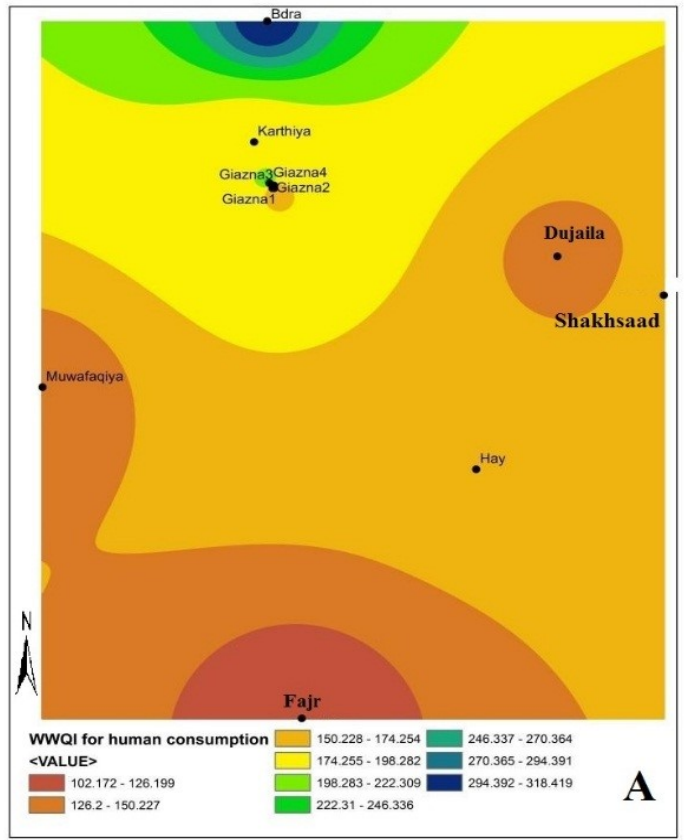

degree of quality of each Parameter, $\mathrm{Ci}=$ concentration size,

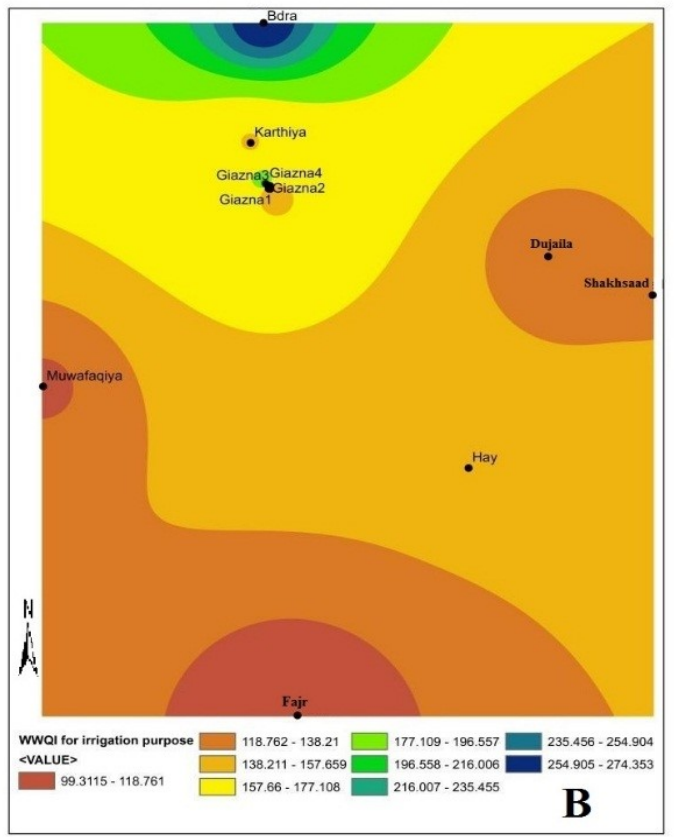

Figure 4: The patterns of distribution of A WWQI for human purposes, B for Irrigation purposes

Table 4-A: Constructed models for SAR prediction of Well waters

\begin{tabular}{|c|c|c|c|c|c|c|c|c|c|}
\hline \multicolumn{2}{|c|}{ Models } & \multirow[b]{2}{*}{ Models } & \multicolumn{7}{|c|}{ Coefficients } \\
\hline & & & $\mathbf{a}_{1}$ & $\mathbf{a}_{1}$ & a3 & $\mathbf{a 4}$ & as & $\mathbf{a}_{6}$ & $\mathbf{a}_{7}$ \\
\hline \multirow{5}{*}{ SAR } & 1 & $\begin{array}{l}\text { SAR=EXP }\left(\mathrm{a}_{1} * \mathrm{Na}^{1=}+\mathrm{a}_{2} * \mathrm{Ca}^{2+}+\mathrm{a}_{3} * \mathrm{Mg}^{2+}+\mathrm{a}\right. \\
\left.4^{*} \mathrm{ESP}+\mathrm{a}_{5}\right)\end{array}$ & $\begin{array}{l}0.0164531771 \\
1\end{array}$ & $\begin{array}{l}- \\
0.007753783 \\
294\end{array}$ & $\begin{array}{l}- \\
0.007788694 \\
031\end{array}$ & $\begin{array}{l}0.521342053 \\
7\end{array}$ & $\begin{array}{l}- \\
3.20539952 \\
5\end{array}$ & - & - \\
\hline & 2 & $\begin{array}{l}\mathrm{SAR}=\mathrm{a}_{1} * \mathrm{Na}^{1+}+\mathrm{a}_{2} * \mathrm{Ca}^{2+}+\mathrm{a}_{3} * \mathrm{Mg}^{2+}+\mathrm{a}_{4} * \mathrm{ES} \\
\mathrm{P}+\mathrm{a}_{5} * \text { Xeast }+\mathrm{a}_{6} * \mathrm{Y} \text { north }+\mathrm{a}_{7}\end{array}$ & $\begin{array}{l}0.0009418399 \\
543\end{array}$ & $\begin{array}{l}- \\
0.000232497 \\
5486\end{array}$ & $\begin{array}{l}- \\
0.000618947 \\
4702\end{array}$ & 1.562783868 & $\begin{array}{l}- \\
2.60190034 \\
\text { E-009 }\end{array}$ & $\begin{array}{l}- \\
4.48342422 \\
1 \mathrm{E}-009\end{array}$ & $\begin{array}{l}-9.79893 \\
7336\end{array}$ \\
\hline & 3 & $\begin{array}{l}\text { SAR }=\operatorname{EXP}\left(a_{1} * E S P+a_{2} * X \text { east }+a_{3} *\right. \\
\left.Y \text { north }+a_{4}\right)\end{array}$ & 0.5556354674 & $\begin{array}{l}- \\
2.704887924 \\
\text { E-008 }\end{array}$ & $\begin{array}{l}- \\
2.549157529 \\
\text { E-007 }\end{array}$ & $2.537384207-$ & - & - & - \\
\hline & 4 & $\begin{array}{l}\mathrm{SAR}=\operatorname{EXP}\left(\mathrm{a}_{1} * \mathrm{Ec}+\mathrm{a}_{2} * \mathrm{pH}+\mathrm{a}_{3} * E S P+\mathrm{a}_{4} *\right. \\
\left.\mathrm{TDS}+\mathrm{a}_{5}\right)\end{array}$ & $\begin{array}{l}1.835844878 \mathrm{E} \\
+013\end{array}$ & $\begin{array}{l}0.012770554 \\
96\end{array}$ & $\begin{array}{l}0.544514501 \\
8\end{array}$ & $\begin{array}{l}- \\
2.868507622 \\
E+010\end{array}$ & $-\overline{3.44780184}$ & - & - \\
\hline & 5 & $\begin{array}{l}\text { SAR }=a_{1} * E c+a_{2} * p H+a_{3} * E S P+a_{4} * T D S+a_{5} \\
X \text { east }+a_{6} * \text { Ynorth }+a_{7}\end{array}$ & $\begin{array}{l}- \\
3.392461612 \mathrm{E} \\
+011\end{array}$ & $\begin{array}{l}- \\
0.000110907 \\
7492\end{array}$ & 1.564115409 & 530072126.8 & $\begin{array}{l}2.09323849 \\
9 \mathrm{E}-\quad 009\end{array}$ & $\begin{array}{l}6.47440633 \\
5 \mathrm{E}-009\end{array}$ & $\begin{array}{l}9.847743 \\
365\end{array}$ \\
\hline
\end{tabular}

Table 4-B: Constructed models for ESP prediction of Wells water

\begin{tabular}{|c|c|c|c|c|c|c|c|c|c|}
\hline \multirow{2}{*}{\multicolumn{2}{|c|}{ Models }} & \multirow[b]{2}{*}{ Models } & \multicolumn{7}{|c|}{ Coefficients } \\
\hline & & & $a_{1}$ & $\mathbf{a}_{1}$ & a3 & $\mathbf{a}_{4}$ & a5 & a6 & $\mathbf{a}_{7}$ \\
\hline \multicolumn{10}{|c|}{ Property } \\
\hline & 1 & $\mathrm{ESP}=\mathrm{a}_{1} * \mathrm{Na}^{1+}+\mathrm{a}_{2} * \mathrm{Ca}^{2+}+\mathrm{a}_{3} * \mathrm{Mg}^{2+}+\mathrm{a}_{4}$ & 0.2589553694 & $\begin{array}{l}- \\
0.07918076287\end{array}$ & $0.0771595101-$ & 7.23198983 & - & - & - \\
\hline & 2 & $\begin{array}{l}\mathrm{ESP}=\operatorname{Exp}\left(\mathrm{a}_{1} * \mathrm{Na}^{1+}+\mathrm{a}_{2} * \mathrm{Ca}^{2+}+\mathrm{a}_{3} * \mathrm{Mg}^{2+}+\mathrm{a}_{4} *\right. \\
\mathrm{X} \text { east }+\mathrm{a}_{5} * \mathrm{Y} \text { north }+\mathrm{a}_{6}\end{array}$ & 0.03270999684 & - & - 0.009830207207 & $\begin{array}{l}3.782628925 \mathrm{E}- \\
008\end{array}$ & $\begin{array}{l}- \\
1.370008817 \mathrm{E}- \\
008\end{array}$ & 2.010132426 & - \\
\hline & 3 & $\begin{array}{l}\mathrm{ESP}=\operatorname{Exp}\left(\mathrm{a}_{1} * E c+a_{2} * X \text { east }+\mathrm{a}_{3} * Y\right. \\
\left.\text { north }+\mathrm{a}_{4}\right)\end{array}$ & 0.03330176606 & $\begin{array}{l}- \\
1.951461554 \mathrm{E}- \\
007\end{array}$ & $3.727132816 \mathrm{E}-008$ & 1.992590805 & - & - & - \\
\hline \multirow[t]{2}{*}{ Esp } & 4 & $\mathrm{ESP}=\mathrm{a}_{1} * \mathrm{Ec}+\mathrm{a}_{2} * \mathrm{PH}+\mathrm{a}_{3} * \mathrm{TDS}+\mathrm{a}_{4}$ & $-\overline{2.630712457 \mathrm{E}+013}$ & 0.2523640582 & $4.110488214 \mathrm{E}+010$ & 5.737897274 & - & - & - \\
\hline & 5 & $\begin{array}{l}\mathrm{ESP}=\mathrm{a}_{1} * \mathrm{Ec}+\mathrm{a}_{2} * \mathrm{pH}+\mathrm{a}_{3} * \mathrm{TDS}+\mathrm{a}_{4} * \mathrm{X} \text { east }+\varepsilon \\
\text { north }\end{array}$ & $1.399566972 \mathrm{E}+013$ & 0.2654706804 & - & $\begin{array}{l}8.55482451 \mathrm{E}- \\
007\end{array}$ & $\begin{array}{l}1.462280765 \mathrm{E}- \\
006\end{array}$ & - & - \\
\hline
\end{tabular}


$\mathrm{Si}=$ value permitted in Iraq. Where the water is classified depending on water quality index (WQI) as shown in Table (2A and 2B) according to House (1989).

Table 5-A: Measured and predicted Values of SAR of studied Well's water and validation indices of constructed models

\begin{tabular}{|c|c|c|c|c|c|c|}
\hline & Property & SAR & & & & \\
\hline \multirow[t]{3}{*}{ Well no. } & Models & 1 & 2 & 3 & 4 & 5 \\
\hline & Measured & 2.824 & 2.824 & 2.824 & 2.824 & 2.824 \\
\hline & predicted & 2.832 & 2.824 & 2.785 & 2.599 & 2.824 \\
\hline \multirow[t]{3}{*}{1} & Residuals & -0.008 & 0.000 & 0.039 & -0.131 & 0.000 \\
\hline & Error\% $\%$ & -0.280 & 0.002 & 1.374 & -4.634 & 0.000 \\
\hline & Measured & 2.675 & 2.675 & 2.675 & 2.675 & 2.675 \\
\hline \multirow[t]{5}{*}{2} & predicted & 2.757 & 2.675 & 2.642 & 2.853 & 2.675 \\
\hline & Residuals & 0.018 & 0.000 & 0.033 & -0.178 & 0.000 \\
\hline & Error\% $\%$ & 0.672 & 0.000 & 1.233 & -6.646 & 0.000 \\
\hline & Measured & 2.855 & 2.855 & 2.855 & 2.855 & 2.855 \\
\hline & predicted & 2.836 & 2.855 & 2.816 & 3.073 & 2.855 \\
\hline \multirow[t]{4}{*}{3} & Residuals & 0.019 & 0.000 & 0.039 & -0.218 & 0.000 \\
\hline & Error $\%$ & 0.651 & -0.004 & 1.363 & -7.620 & 0.000 \\
\hline & Measured & 2.584 & 2.584 & 2.584 & 2.584 & 2.584 \\
\hline & predicted & 2.545 & 2.585 & 2.555 & 2.733 & 2.584 \\
\hline \multirow[t]{4}{*}{4} & Residuals & 0.039 & -0.001 & 0.029 & -0.149 & 0.000 \\
\hline & Error\% $\%$ & 1.495 & -0.021 & 1.125 & -5.758 & 0.000 \\
\hline & Measured & 3.415 & 3.415 & 3.415 & 3.415 & 3.415 \\
\hline & predicted & 3.403 & 3.415 & 3.438 & 3.824 & 3.416 \\
\hline \multirow[t]{4}{*}{5} & Residuals & 0.012 & 0.000 & -0.023 & -0.409 & -0.001 \\
\hline & Error $\%$ & 0.360 & -0.002 & -0.662 & -11.973 & -0.022 \\
\hline & Measured & 2.297 & 2.297 & 2.297 & 2.297 & 2.297 \\
\hline & predicted & 2.311 & 2.297 & 2.312 & 2.459 & 2.297 \\
\hline \multirow[t]{3}{*}{6} & Residuals & -0.014 & 0.000 & -0.015 & -0.162 & 0.000 \\
\hline & Error $\%$ & -0.592 & 0.021 & -0.666 & -7.063 & 0.000 \\
\hline & Measured & 2.415 & 2.415 & 2.415 & 2.415 & 2.415 \\
\hline \multirow[t]{4}{*}{7} & predicted & 2.405 & 2.415 & 2.420 & 2.587 & 2.416 \\
\hline & Residuals & 0.01 & 0.000 & -0.005 & -0.172 & -0.001 \\
\hline & Error\% $\%$ & 0.420 & -0.017 & -0.218 & -7.137 & -0.026 \\
\hline & Measured & 2.104 & 2.104 & 2.104 & 2.104 & 2.104 \\
\hline \multirow[t]{4}{*}{8} & predicted & 2.158 & 2.104 & 2.171 & 2.274 & 2.105 \\
\hline & Residuals & -0.054 & 0.000 & -0.067 & -0.170 & -0.001 \\
\hline & Error $\%$ & -2.587 & -0.007 & -3.181 & -8.100 & -0.031 \\
\hline & Measured & 2.435 & 2.435 & 2.435 & 2.435 & 2.435 \\
\hline \multirow[t]{5}{*}{9} & predicted & 2.421 & 2.434 & 2.444 & 2.587 & 2.434 \\
\hline & Residuals & 0.014 & 0.001 & -0.009 & -0.152 & 0.001 \\
\hline & Error $\%$ & 0.590 & 0.025 & -0.359 & -6.257 & 0.030 \\
\hline & Measured & 3.671 & 3.671 & 3.671 & 3.671 & 3.671 \\
\hline & predicted & 3.695 & 3.671 & 3.691 & 4.233 & 3.671 \\
\hline \multirow[t]{3}{*}{10} & Residuals & -0.024 & 0.000 & -.020 & -0.562 & 0.000 \\
\hline & Error & -0.647 & 0.004 & --0.554 & -15.299 & 0.000 \\
\hline & Measured & 2.799 & 2.799 & 2.799 & 2.799 & 2.799 \\
\hline \multirow[t]{10}{*}{11} & predicted & 2.813 & 2.799 & 2.803 & 2.920 & 2.799 \\
\hline & Residuals & -0.014 & 0.000 & -0.004 & -0.121 & 0.000 \\
\hline & Error $\%$ & -0.517 & 0.000 & -0.145 & -4.338 & 0.000 \\
\hline & coefficient of determination $R^{2}$ & 0.996 & 0.9999 & 0.9950 & 0.997 & 0.999 \\
\hline & SEE & 3.32 & 5.30 & 3.91 & 3.20 & 6.42 \\
\hline & RMSE & 0.025 & 0.000 & 0.031 & 0.257 & 0.000 \\
\hline & $M A E$ & 0.021 & 0.000 & 0.026 & 0.220 & 0.000 \\
\hline & $R E$ & 0.008 & 0.000 & 0.009 & 0.075 & 0.000 \\
\hline & $S$ & 0.036 & 0.0004 & 0.2683 & 0.2044 & 0.0006 \\
\hline & $r$ & 0.997 & 0.9999 & 0.7785 & 0.9475 & 0.999 \\
\hline
\end{tabular}

\section{Model development and construction:}

Data Fit software (ver. 9.1.32) was used to construct the mathematical models required to predict the values of SAR
Table 5 -B: Measured and predicted Values of ESP of studied Well's water and validation indices of constructed models

\begin{tabular}{|c|c|c|c|c|c|c|}
\hline \multirow[b]{2}{*}{ Well no. } & \multicolumn{2}{|l|}{ Property } & \multicolumn{3}{|c|}{ ESP } & \multirow[b]{2}{*}{5} \\
\hline & Models & 1 & 2 & 3 & 4 & \\
\hline \multirow{5}{*}{1} & Measured & 8.087 & 8.087 & 8.087 & 8.087 & 8.087 \\
\hline & predicted & 8.070 & 8.068 & 7.841 & 7.933 & 8.046 \\
\hline & Residuals & 0.017 & 0.019 & 0.246 & 0.154 & 0.041 \\
\hline & Error $\%$ & 0.206 & 0.240 & 3.040 & 1.902 & 0.509 \\
\hline & Measured & 7.992 & 7.992 & 7.992 & 7.992 & 7.992 \\
\hline \multirow[t]{5}{*}{2} & predicted & 7.991 & 7.983 & 7.947 & 7.964 & 8.096 \\
\hline & Residuals & 0.001 & 0.009 & 0.045 & 0.028 & -0.104 \\
\hline & Error $\%$ & 0.019 & 0.110 & 0.568 & 0.345 & -1.307 \\
\hline & Measured & 8.107 & 8.107 & 8.107 & 8.107 & 8.107 \\
\hline & predicted & 8.215 & 8.210 & 8.134 & 8.121 & 8.245 \\
\hline \multirow[t]{4}{*}{3} & Residuals & -0.108 & -0.103 & -0.027 & -0.014 & -0.138 \\
\hline & Error $\%$ & -1.334 & -1.270 & -0.331 & -0.169 & -1.708 \\
\hline & Measured & 7.934 & 7.934 & 7.934 & 7.934 & 7.934 \\
\hline & predicted & 7.898 & 7.886 & 8.082 & 7.714 & 7.833 \\
\hline \multirow[t]{4}{*}{4} & Residuals & 0.036 & 0.048 & -0.148 & 0.20 & 0.101 \\
\hline & Error\% & 0.450 & 0.602 & -1.865 & 2.76 & 1.274 \\
\hline & Measured & 8.466 & 8.466 & 8.466 & 8.466 & 8.466 \\
\hline & predicted & 8.450 & 8.451 & 8.354 & 8.285 & 8.417 \\
\hline \multirow[t]{4}{*}{5} & Residuals & 0.016 & 0.015 & 0.112 & 0.181 & 0.049 \\
\hline & Error $\%$ & 0.192 & 0.174 & 1.319 & 2.141 & 0.575 \\
\hline & Measured & 7.750 & 7.750 & 7.750 & 7.750 & 7.750 \\
\hline & predicted & 7.758 & 7.761 & 7.895 & 7.652 & 7.779 \\
\hline \multirow[t]{3}{*}{6} & Residuals & -0.008 & -0.011 & -0.145 & 0.098 & -0.029 \\
\hline & Error\% & -0.100 & -0.145 & -1.872 & 1.265 & -0.372 \\
\hline & Measured & 7.826 & 7.826 & 7.826 & 7.826 & 7.826 \\
\hline \multirow[t]{4}{*}{7} & predicted & 7.813 & 7.833 & 7.855 & 7.714 & 7.864 \\
\hline & Residuals & 0.013 & -0.007 & -0.029 & 0.112 & -0.038 \\
\hline & Error $\%$ & 0.160 & -0.093 & -0.373 & 1.425 & -0.488 \\
\hline & Measured & 7.626 & 7.627 & 7.627 & 7.627 & 7.627 \\
\hline \multirow[t]{4}{*}{8} & predicted & 7.657 & 7.650 & 7.933 & 7.644 & 7.699 \\
\hline & Residuals & -0.030 & -0.023 & -0.306 & -0.017 & -0.072 \\
\hline & Error $\%$ & -0.396 & -0.302 & -4.007 & -0.225 & -0.942 \\
\hline & Measured & 7.838 & 7.838 & 7.838 & 7.838 & 7.838 \\
\hline \multirow[t]{5}{*}{9} & predicted & 7.828 & 7.825 & 7.962 & 7.714 & 7.806 \\
\hline & Residuals & 0.014 & 0.013 & -0.124 & 0.124 & 0.032 \\
\hline & Error $\%$ & 0.176 & 0.160 & -1.581 & 1.57 & 0.403 \\
\hline & Measured & 8.629 & 8.629 & 8.629 & 8.629 & 8.629 \\
\hline & predicted & 8.613 & 8.616 & 8.557 & 8.347 & 8.606 \\
\hline \multirow[t]{3}{*}{10} & Residuals & 0.016 & 0.013 & 0.072 & 0.282 & 0.023 \\
\hline & Error & 0.184 & 0.155 & 0.838 & 3.265 & 0.262 \\
\hline & Measured & 8.071 & 8.071 & 8.071 & 8.071 & 8.071 \\
\hline \multirow[t]{10}{*}{11} & predicted & 8.037 & 8.043 & 7.767 & 7.918 & 7.954 \\
\hline & Residuals & 0.037 & 0.028 & 0.304 & 0.153 & 0.117 \\
\hline & Error $\%$ & 0.418 & 0.346 & 3.764 & 1.901 & 1.445 \\
\hline & coefficient of determination $R^{2}$ & 0.9818 & 0.9827 & 0.633 & 0.924 & 0.924 \\
\hline & SEE & 4.81 & 5.55 & 0.22 & 9.83 & 0.11 \\
\hline & RMSE & 0.038 & 0.037 & 0.172 & 0.150 & 0.078 \\
\hline & MAE & 0.027 & 0.026 & 0.142 & 0.126 & 0.068 \\
\hline & $R E$ & 0.003 & 0.003 & 0.018 & 0.016 & 0.008 \\
\hline & S & 0.3518 & 0.041 & 0.1582 & 0.0777 & 0.0824 \\
\hline & $R$ & 0.5153 & 0.991 & 0.806 & 0.956 & 0.962 \\
\hline
\end{tabular}


and ESP for the well waters. The Model building and the choice of the chemical attributes were accomplished depending on the strength of the inter-relations among the water chemical parameters. Different combinations of spatial and chemical attributes were used to develop the models. As a result, five models for predicting SAR and ESP values were constructed. Consequently, ten models were built, as listed in Table 4 (4A and 4B). The sequence of working steps of model building is as follows:

Start $>$ Worksheet $>$ Input data $>$ solve $>$ Regression $>$ nonlinear $>$ model choice $>$ fit information

The nonlinear regression analysis was adopted, and the models were validated using $\mathrm{R}^{2}$, RMSE, MAE, and RE, Table (5A and 5B). These indices were computed using formulas 8,9 , and 10 , respectively.

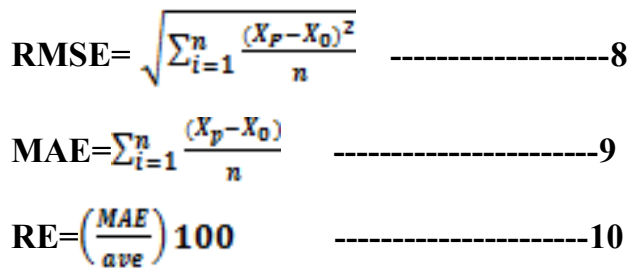

Where $\left(\mathrm{x}_{\mathrm{o}}\right),\left(\mathrm{x}_{\mathrm{p}}\right),(\mathrm{n}),($ ave) are observed and predicted values, the number of samples, and the mean value of predicted values, respectively.

Besides, the accuracy of models used in predicting SAR and ESP values was tested by comparing the measured and estimated values of SAR and ESP using Curve Expert software 1.4 (1995); Table 5A, 5B and Figure $(6 a, 6 b, 6 c, 6 d)$. The sequence steps of using the program are as follows:

Start $>$ New $>$ worksheet $>$ Data input $>$ Linear $>$ Apply fit

\section{Results and Discussion}

\section{Descriptive statistics}

Table (1) presents a summary of the descriptive statistical analysis carried out on the chemical properties of well waters. The minimum and maximum values of $\mathrm{Na}^{1+}$ were ranging from 4.783 to 13.391 meq $\mathrm{L}^{-1}$ for Muwafaqiya and Badra wells, respectively, with mean equals $7.221 \mathrm{meq} \mathrm{L}^{-1}$. Whereas $\mathrm{Ca}^{2+}$ values ranged between $3.735 \mathrm{meq} \mathrm{L}^{-1}$ and 16.504 meq $\mathrm{L}^{-1}$ with mean equals $9.031 \mathrm{meq}^{-1}$ in Fajr and Badra well waters, respectively. The minimum and maximum $\mathrm{Mg}^{2+}$ values were $2.332 \mathrm{meq} \mathrm{L}^{-1}$ and $11.532 \mathrm{meq} \mathrm{L}^{-1}$ in the water samples of Muwafaqiya and Giazna 4, respectively. EC and TDS values ranged from $1.200 \mathrm{dS} \mathrm{m}^{-1}$ to $4.00 \mathrm{dS} \mathrm{m}^{-1}$ with mean $2.170 \mathrm{dS} \mathrm{m}^{-1}$ and from $768.000 \mathrm{mg} \mathrm{L}^{-1}$ to
$2560.0 \mathrm{mg} \mathrm{L}^{-1}$ with mean $1391.180 \mathrm{mg} \mathrm{L}^{-1}$ for Muwafaqiya and Badra well waters, respectively. The min. and max. values of $\mathrm{pH}$ were 6.25 and 7.80 , respectively, for well waters of Karthiya, Giazna 4 and Fajr with mean 7.11. The SAR and ESP min. and max. values were (2.104 - 3.671) and (7.627-8.629), respectively, for Muwafaqiya and Badra with means equal 2.734 and 8.030 for SAR and ESP. Additionally, the spatial pattern of distribution of these chemical attributes for the studied wells was shown in Figure 2. The high values of ions and TDS in well waters of the study area and their spatial pattern of distribution was related to the amounts of major element dissolved in the underground water and due to human activities besides the evaporation which leads to raise the concentration of these ions and finally to the seasonal fluctuation of rain (Faridabad, 2010; Abdel-Aziz, 2018).

\section{Spatial variability in chemical properties of well waters}

Table (1A and $1 \mathrm{~B})$ presents the spatial variability of chemical parameters of wells groundwater in the study area. The skewness coefficient was used to characterize the normality of the distribution of these parameters. The positive skewness coefficient ranged from 0.728 to 1.664 for $\mathrm{Ca}^{2+}$ and $\mathrm{Mg}^{2+}$, respectively, and the only negatively skewed property is $\mathrm{pH}(-0.209)$. The results showed highly skewed distributions except for $\mathrm{pH}$ and $\mathrm{Ca}^{2+}$ which were with symmetrical and moderately skewed distributions, respectively, and of the platykurtic shape of a distribution, Brown (2014). This may be due to the calcareousness of the soil which water of the studied wells passes through; the major elements in the groundwater express the intensity of water-rock interface (Elhag, 2016). The pattern of spatial variability for the chemical properties of the studied well waters has the following form of sequence:

$\mathrm{Mg}^{2+}>\mathrm{EC}, \mathrm{TDS}>\mathrm{Na}^{1+}>\mathrm{Ca}^{2+}>\mathrm{SAR}>\mathrm{pH}>\mathrm{ESP}$.

The coefficient of variation $(\mathrm{Cv} \%)$ ranges between 3.71 and 69.240 for ESP and $\mathrm{Mg}^{2+}$ and with standard deviations 0.298 and 3.302, respectively. ESP and $\mathrm{pH}$ are classified of low variability and SAR of moderate one; however, $\mathrm{Ca}^{2+}, \mathrm{Na}^{1+}, \mathrm{EC}$, TDS and $\mathrm{Mg}^{2+}$ are highly variable attributes. This variation may be inferred to the geological characteristics of aquifers (Elhag, 2016), and the fluctuations of rainwater. The spatial variability of studied chemical properties of well waters is illustrated with the form of histograms depicted in Figure 3 (A, B, C, $\mathrm{D}, \mathrm{E})$. Figure 3 shows that the chemical properties varied according to their geographical location and according to 
human, agricultural and anthropogenic processes and also to the seasonal variability of rainfall.

\section{Well waters quality index (WWQI)}

Table (2A and 2B) presents quality indices of well waters for human and irrigation purposes. The indices of $\mathrm{pH}$
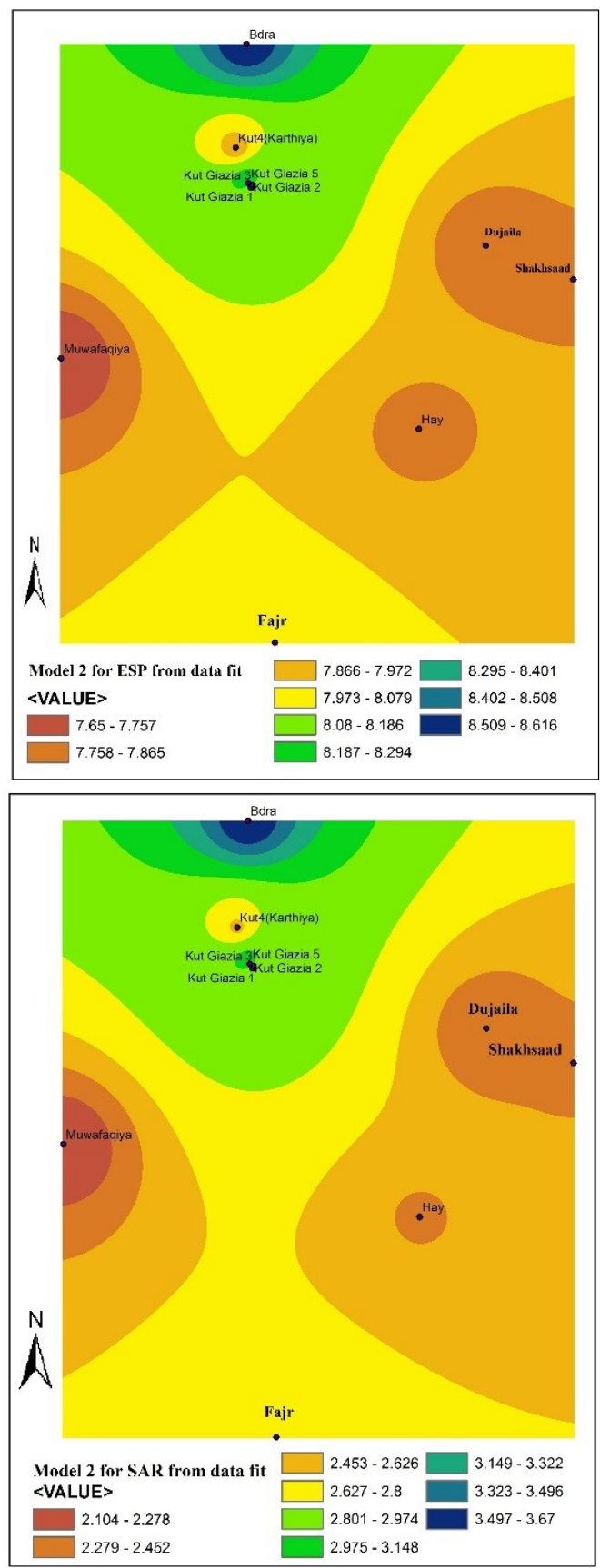

range between 11.11 and 13.87 in Karthiya and Fajr, Giazna 4 wells. EC quality index shows relatively high figures and ranged from 120 to $400 \mathrm{dS} \mathrm{m}^{-1}$, in Badra and Fajr wells. Whilst, TDS quality index ranged from 0.08 to 0.26 for well waters of Fajr and Badra. Also, Muwafaqiya and Badra wells revealed $\mathrm{Na}^{1+}$ quality index of 6.33 and 17.71 , respectively.
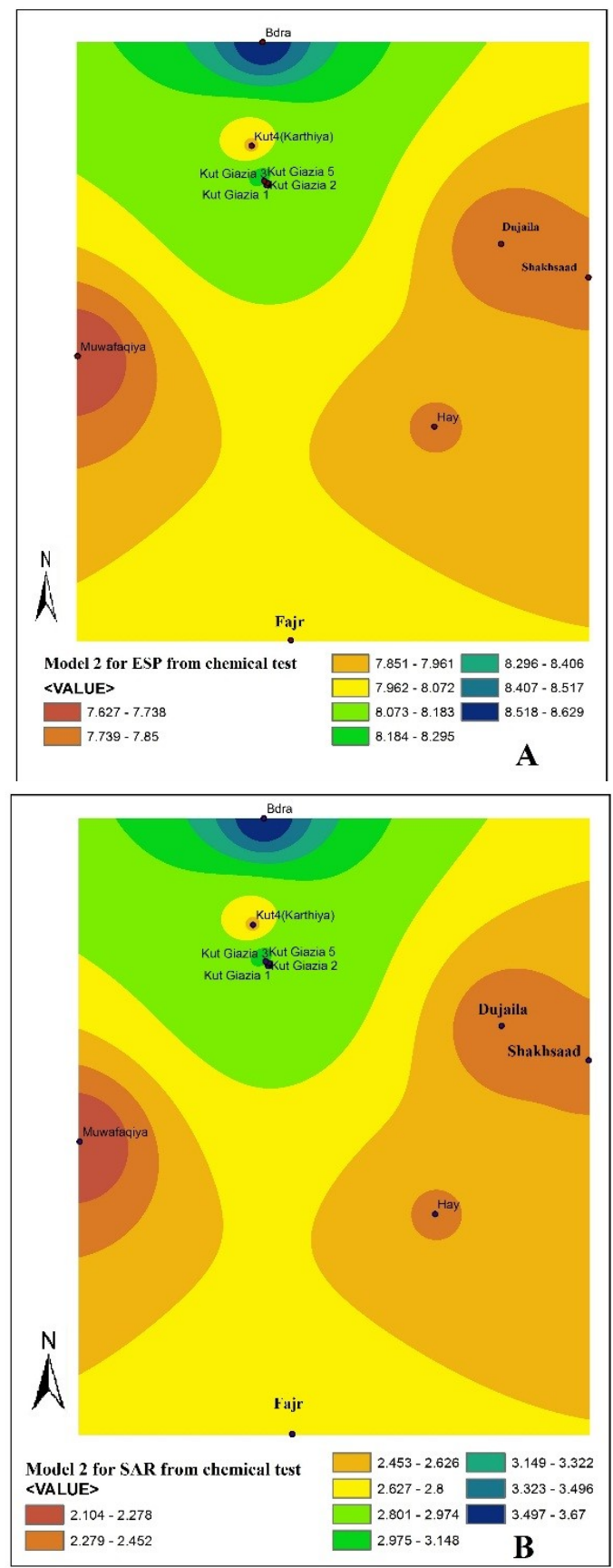

Figure 5 (A): ESP before and after modelling, (B): SAR before and after modelling 
Whereas $\mathrm{Ca}^{2+}$ and $\mathrm{Mg}^{2+}$ ions showed quality index ranging between 3.73 to 16.5 and 13.44 to 66.48 for Fajr, Badra, Muwafaqiya and Geazna 4 wells, respectively. However, SAR and ESP indices for irrigation purposes ranged between 19.68 to 34.33 and 10.89 to 12.32 , respectively, in Muwafaqiya and Badra. These variations in chemical attributes quality indices are due to the geological and chemical characteristics of the aquifer (Elhag, 2016). Moreover, the sequence of the lowest chemical parameter's quality indices for human and irrigation purposes was found taking the following pattern: TDS $<\mathrm{Ca}^{2+}<$ $\mathrm{Na}^{1+}<\mathrm{pH}<\mathrm{Mg}^{2+}<\mathrm{EC}$. As a result, the WWQI of well waters for human purpose ranged between 102.17 and 318.500 in Fajr and Badra wells, respectively. The sequence of WWQI for human purposes took the following pattern: Fajr $<$ Giazna $1<$ Muwafaqiya $<$ Dujaili $<$ Giazna $2<$ Shakhsaad $<$ Hay $<$ Karthiya $<$ Giazna $3<$ Badra. And for irrigation purposes were between 99.310 and 274.420 in Fajr and Badra wells, respectively. These high WWQ indices in these areas are due to the degradation of groundwater by natural and anthropogenic processes (Barzegar and Moghaddam, 2016). The sequence of the lowest quality index of chemical attributes for irrigation purposes was as follows: Fajr $<$ Giazna $1<$ Muwafaqiya $<$ Dujaili $<$ Giazna $2<$ Shaikhsaad $<$ Hay $<$ Karthiya $<$ Giazna 3 $<$ Giazna $4<$ Badra. The geographic patterns of distribution of WWQI is illustrated in Figure 4 ( $\mathrm{A}$ and $\mathrm{B}$ ) by using GIS technique. Hence, all waters of the study area are classified as unsuitable for both human and irrigation purposes except for well Fajr that was classified critically and marginally suitable for irrigation purposes and should be carefully used with the association of good soil management practices. The high WWQI figures of the water of the studied wells may be due to the agricultural activities, seasonal fluctuations of rain, various contaminations caused by Anthropogenic activities and the improper waste disposal which rendered the water to be unsuitable quality (AbdEl-Aziz, 2018; Ferchichi, et al. 2018).

\section{The inter-relations between chemical properties of well waters}

Table (3) presents the inter-relations between studied parameters of well waters employing correlation coefficients using two-tailed Pearson correlation. Most of the attributes showed a highly positive significant correlation $(\rho=0.01$ and $\rho=0.05)$. The highest positive correlation was between EC and TDS $\left(1.00^{* *}\right)$ followed by $\left(0.999^{* *}\right)$ between SAR and ESP. Whereas the lowest positive correlation coefficients were 0.218 and 0.232 between $\mathrm{pH}, \mathrm{Y}$ (northing) coordinates, EC and TDS, respectively. Whereas, the chemical properties were negatively correlated with $\mathrm{X}$ (Easting spatial coordinates), $(\mathrm{r}$ $=-0.009$ and -0.171 ) between TDS, EC and $\mathrm{Mg}^{2+}$. In contrast, most of the studied chemical properties revealed highly significant positive correlation coefficients that ranged between $0.663^{*}$ and $0.800^{* *}$ with $\mathrm{Y}$ (northing) coordinates. Accordingly, these relations were used to choose the appropriate properties to develop mathematical models to predict the SAR and ESP of well waters.

\section{Predicting ESP and SAR values of well waters and mathematical models}

Table 4A and 4B present the constructed mathematical models used for estimating SAR and ESP values of well waters. Different combinations of chemical properties of well waters were used to develop the mathematical models, Table (4A and 4B). The models were evaluated according to their highest value of $\mathrm{R}^{2}$ and lowest SEE value that resulted from the model fit information and the lowest calculated values of RMSE, RE, and MAE. Consequently, ten models were developed. Five models for SAR and five models for ESP. Accordingly, Model 2 was the best in predicting values of SAR and ESP of studied well waters. The lowest and highest positive and negative error percentages of model 2 were ranging from 0.000 to 0.025 and from -0.002 and -0.021 in Giazna 2, Fajr, Hay, Giazna 4 and Karthiya wells, respectively. Model 2 had 0.000 residuals in predicting the SAR value of most well waters except in Hay and Karthiya wells, where the residuals were +0.001 and -0.001 , respectively. The positive and negative error percentages and residuals of model 2 used for prediction of ESP values were ranging from 0.11 to 0.602 ; from -0.093 to -1.27 ; from +0.009 to 0.048 ; and from -0.007 to -0.103 in the wells of Giazna 2, Karththiya, Shaikhsaad, and Giazna 3, respectively.

\section{Validation and accuracy of developed models}

The developed models were tested for their accuracy and validity. The testing was done utilizing $\mathrm{R}^{2}$, RMSE, MAE, AE, $s$, and $r$ indices for the predicted values of SAR and ESP of studied well waters; relevant details are shown in Table 5A, $5 \mathrm{~B}$ and Figures 5A, 5B, 6A, 6B, 6C, and 6D. The determination coefficients of models used in predicting SAR values ranged from 0.995 to 0.999 in models 3, 2, and 5, respectively, and its range in predicting ESP values are between 0.633 and 0.9827 for models 3 and 2, respectively. The other indices ranges used in assessing the estimating of SAR were SEE (3.200 - 6.420) in models 4 and 5, respectively, RMSE (0.000 -0.257) in models 2, 5 and 4 , respectively, MAE (0.000-0.220) in models 2, 5 and 4 , respectively, RE $(0.000-0.075)$ in models 2,5 , and 4 , respectively, s $(0.0004-0.2683)$ in models 2 and 3 , respectively, and $\mathrm{r}(0.778-0.999)$ in models 3,2 and 5, respectively. In contrast, the ranges of SEE, RMSE, MAE, $\mathrm{RE}, \mathrm{s}$, and $\mathrm{r}$ for the models used in estimating ESP value were: SEE (0.11-9.83) in models 5 and 4; RMSE (0.037- 
0.172) in models 2 and 3, respectively, MAE (0.026 - 0.142) in models 2 and 3, respectively, RE (0.003 - 0.018) in models 1,2 and 3, respectively, $\mathrm{S}(0.041-0.3518)$ in models 2 and 1, respectively, $\mathrm{r}(0.5153-0.991)$ in models 1 and 2 , respectively. Consequently, model 2 is the best model among all the models developed to estimate SAR and ESP values for the studied well waters. This was followed by model 5 , as shown in Table 5A, 5B and in Figures 5A, 5B, 6A, 6B, 6C, and $6 \mathrm{D}$. That is due to their high $\mathrm{R}^{2}$ values and their very low RMSE, SEE, MAR, RE, and s values comparing with other models. This conclusion was confirmed by the result of $s$ and $\mathrm{r}$ computed using CurveExpert software (ver.1.4, 1995), Figure 6 (A, B, C, and D.).

\section{Conclusions}

Groundwater is a vital and effective source of water in arid and semi-arid regions, and thus monitoring its quality is very essential to develop effective strategies for improving rural drinking water and helping in making decision making regarding its management. Mathematical modelling helped predict the SAR and ESP values of groundwater. The analysis showed a great advantage in estimating SAR and ESP values. Model 2 was the best and yielded very close values of SAR and ESP of well waters compared to the chemically measured values.

Chemical properties of well waters were spatially

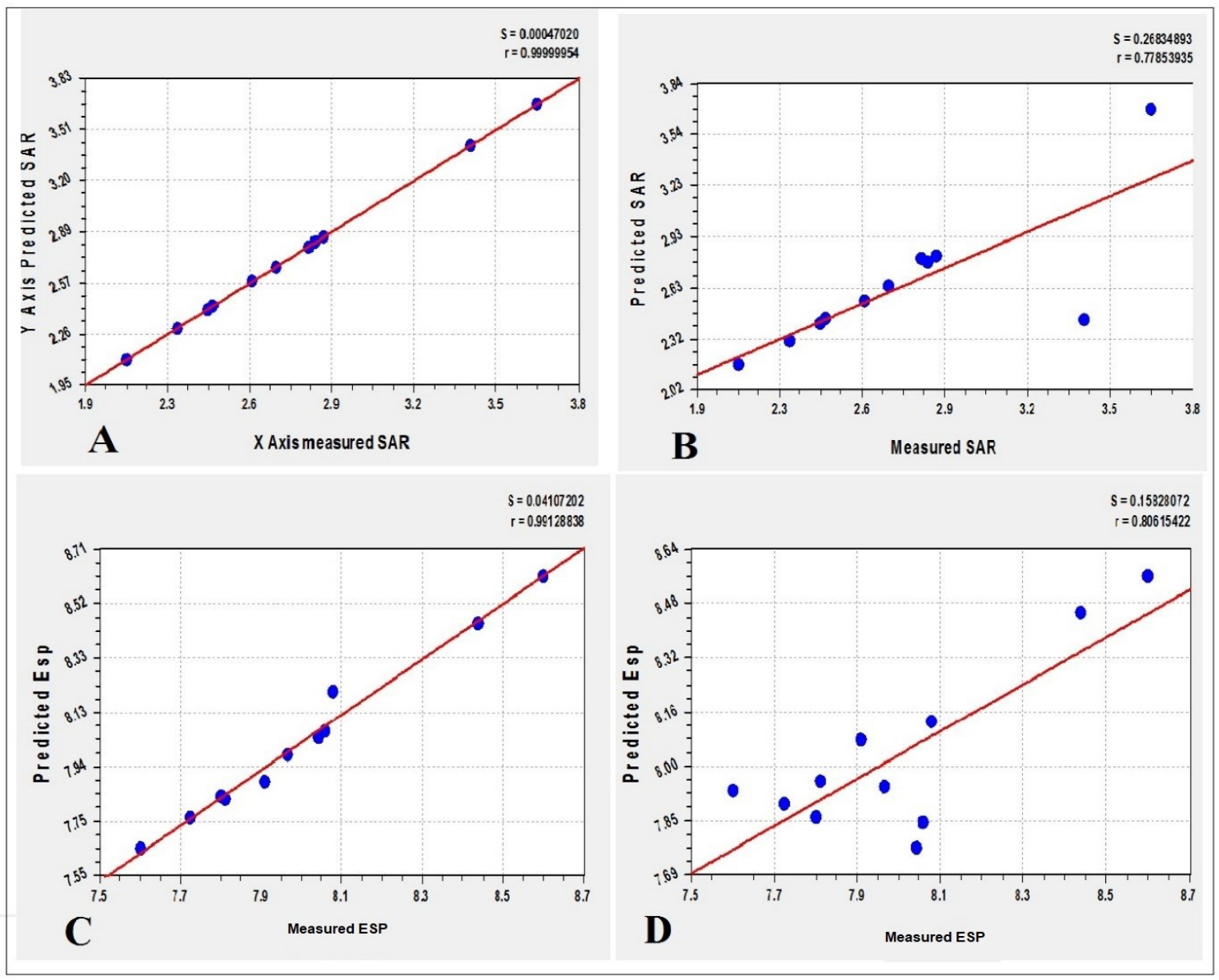

Figure (6): A -The relationship between measured and predicted SAR of studied well waters-model 2. B- The relationship between measured and predicted SAR of studied well waters-model $-\mathbf{3}$. C- The relationship between measured and predicted ESP of studied well waters - model 2. D-The relationship between measured and predicted ESP of studied well waters -model -3 
variable and dependent especially upon easting direction $(\mathrm{X})$ except with $\mathrm{pH}$. The most influential attribute was $\mathrm{Mg}^{2+}$ and the least one was ESP. Besides, the results showed that all well waters were not suitable for both irrigation and human uses except for the well located in Fajr district which was marginally suitable for irrigation uses only and must be used with caution as it would cause a salinity problem for soil. Accordingly, these well waters have to be refined and purified before adopting them for human uses; otherwise, it might cause severe health problems. The accuracy and validity of the developed models can be assured from the values of $s$ and $r$ of model 2 in both SAR and ESP (Table 5A) and GIS maps of their patterns of spatial distribution. Finally, it is possible to conclude that using mathematical models can be useful in predicting SAR and ESP values adequately.

\section{References}

Abbasnia, A., N. Yousefi, A.H. Madhavi, R. Nabizadeh, M. Radford, M. Yousefi and M. Alimohammedi. 2018. Evaluation of groundwater quality and its suitability for assessing water for drinking and irrigation purposes: A case study of Sistan and Baluchistan province (IRAN). Human and Ecological Risk Assessment: An International Journal 25(4): 988-1005.

AbdEl-Aziz, S.H. 2018. Application of traditional method and water Quality Index to assess suitability of groundwater for drinking and irrigation purposes in South-Western region of Libya. Water Conservation and Management 2(2): 20-30.

Abu Sharar, T. 1979. The relation between sodium adsorption ratio (SAR) and exchangeable sodium percentage (ESP) in different Iraqi soils. State Organization of Soil and Land Reclamation. Technical Bull. No.14. p.124. In: Soil salinity, The Theoretical and Applied Principles. A.H. AL Zubaidi (ed.) Dar AL Hikmah, Baghdad University, Ministry of Higher Education and Scientific Research, Baghdad, Iraq.

Aghazadeh, N. and A.A. Moghaddam. 2010. Assessment of groundwater quality and its suitability for drinking and agricultural uses in the Oshnavieh area, Northwest of Iran. Journal of Environmental Protection 1(1): 30-40.

ALsaffawi, Y.T.A, M.A. Abdulhafedh and M.K. ALTaay. 2018. Assessment of Water quality in Mosul university by using the WQI model. Kirkuk University Journal for Scientific Studies 13(2): 185-198.

Barzegar, R. and A.A. Moghaddam. 2016. Combining the advantage of neural networks using the concept of committee machine in the groundwater salinity prediction. Modeling Earth Systems and Environment 2(1): 1-13.
Bhat, M.A., M.S. Grewal, R. Rajpaul, S.A. Wani and E.A. Dar. 2016. Assessment of groundwater quality for irrigation purposes using chemical indices. Indian Journal of Ecology 43(2):574-579.

CurveExpert software version 1.4.1995. A curve fitting system for windows Duple precision.?32-bite package. Tennessee at Chattanooga, USA.

Brown, S. 2014. Measures of shape: Skewness and Kurtosis (math 200) (TC3 Brown). BrownMath.Com. Oakroadsystem.com, New York, USA.

DataFit 9.1.32 (1995-2014) Data engineering software

Durgadevagi, S., R. Annadural and M. Meenu. 2016. Erode, Tamil Nadu, India. Indian Journal of Science and Technology 9(23).

Elhag, A.B. 2016. New diagram useful for classification of groundwater quality. British Journal of Earth Science Research 4(4): 49-54.

Fadil, S.H. and S.R. Majeed. 2009. Studying the validity of the underground water for civil uses (drinking and housing) in Fallujah city. Journal of the University of Anbar for Pure Science 3(2): 99-103.

Faridabad, 2010. Groundwater quality in shallow aquifers of India. Central Groundwater Board. Ministry of Water Resources, Government of India. Faridabad.

Faozi, M.O.K. and F. Aziz. 2006. Characteristics of shwerat area south of mosul, the centre of remote sensing. University of Mosul, Iraq. Journal of Tikrit Pure Science 11(1): 198-202.

Hassan, W.F. and A.A. Mohammed. 2005. Groundwater in southern Iraq. Marin Science Centre - Basrah University. Journal of Basrah Research 1(31).

House, M.A., 1989. Water quality indices as indicators of change. Environmental Monitoring and Assessment 15(3): 255.

Hussain, M., M.W. Muataz, S.M. Hussain, M.N. Abass, S. Mehmood and M. Imran. 2015. Comparative physiochemical characterization and spatial distribution of pollutants in rural and urban drainage water. Soil and Environment 34(1): 51-64.

Khalid, S. 2019. An assessment of groundwater quality for irrigation and drinking purposes around brick Kilns in three districts of Baluchistan province, Pakistan, through water quality index and multivariate statistical approach. Journal of Geochemical Exploration 179: 142615.

Khan, A. and Y. Rehman. 2017. Groundwater quality assessment using water quality index (WQI) in Liaquatabad Town, Karachi, Pakistan. Academic Journal of Environmental Science 5(6): 095-101.

Moghbel, F., B. Mustafazadeh-Fard, A.M.M. Maibody and L. Esmaeil. 2017. Salinity management for irrigation 
with saline-sodic water under corn cultivation. Soil and Environment 36(2): 120-130.

Mohamed, A.K., L. Dan, S. Kia, A.A. Mohamed, E. Eldaw, and B.A. Elubid. 2019. Hydrological analysis and fuzzy logic method for evaluation of groundwater quality in the North Chengdu Plain, China. International Journal of Environmental Research and Public Health 16(3): 302.

Mohammed, A.K., L. Dan, S. Kai, E. Eldaw and S. Abualela. 2019. Evaluating the suitability of groundwater for drinking purposes in North Chengdu, China. The International Symposium on Water Recourses and Environmental Management (WERM) E3S Web of Science 2019 - e3s-conferences.org. Chengdu Plain, China.

Narsimha, A., S. Venkatayogi and S. Geeta. 2018. Hydrogeochemical data on groundwater quality with special emphasis of fluoride enrichment in Munneru river basin (MRB, Telangana State, State, South India. Data in Brief 17: 339-346.

Richard, L.A. 1954. Diagnosis and Improvement of Saline and Alkali Soils. United States Department of Agriculture, Agriculture Handbook No. 60. Govt. Print. Office, Washington, DC, pp 89-106.
Sabeen, M., Q. Mahmood, A. Ebadi, Z.A. Bhatti, F.M. Irshad, A. Kakar, M. Bilal, H.M. Arshad and N. Shahid. 2020. Health risk assessment consequent to wastewater irrigation in Pakistan. Soil Environment 39(1): 67-76. DOI: $10.25252 / \mathrm{SE} / 2020 / 71758$.

SPSS. 2010. IBM *statistical program for social sciences *statistics version 20. New York, USA.

Wali, S., J.K. Umar, S.D. Abdulbaker, I.M. Dankanl and G.Y. Safiyanu. 2018. Variability in aquifer depths dominate physiochemical composition of groundwater in highland area of South-Eastern Sokoto Basin, Nigeria. Journal of Environmental Science and Earth Science 1(2): 1023.

Yasin, G. 2009. Survey of groundwater and surface water quality in AL-Khadimiya town. Journal of Engineering and Technology 27(15): 539-556. 